\title{
Singularities in scalar-tensor gravity
}

\author{
Valerio Faraoni \\ Physics Department, University of Northern British Columbia \\ 3333 University Way, Prince George, B.C., Canada V2N $4 Z 9$
}

\begin{abstract}
The analysis of certain singularities in scalar-tensor gravity contained in a recent paper is completed, and situations are pointed out in which these singularities cannot occur.
\end{abstract}


Recently, Abramo et al. [1] have investigated certain geometric singularities occurring in the gravitational sector of scalar-tensor theories described by the action

$$
S=\int d^{4} x \sqrt{-g}\left[\frac{f(\phi)}{2} R-\frac{\omega(\phi)}{2} g^{a c} \nabla_{a} \phi \nabla_{c} \phi-V(\phi)\right] .
$$

Their analysis generalizes to scalar-tensor gravity previous studies of nonminimally coupled scalar field theory. It is customary to interpret

$$
G_{e f f}(\phi) \equiv \frac{1}{8 \pi f(\phi)}
$$

as an effective gravitational coupling. However, in the weak field limit, the effective coupling measured in a Cavendish experiment assumes the different form $[3,4]$

$$
G_{e f f}^{*}(\phi)=\frac{2 \omega f+(2 d f / d \phi)^{2}}{8 \pi f\left[2 \omega f+3(d f / d \phi)^{2}\right]} .
$$

Both effective couplings diverge at the zeros of $f(\phi)$ and change sign when $\phi$ crosses a value corresponding to a zero of $f$. Moreover, the conformal transformation to the Einstein frame

$$
\begin{gathered}
g_{a b} \longrightarrow \tilde{g}_{a b}=\sqrt{f(\phi)} g_{a b}, \\
\tilde{\phi}=\int \frac{d \phi}{f(\phi)} \sqrt{f(\phi)+\frac{3}{2}\left(\frac{d f}{d \phi}\right)^{2}},
\end{gathered}
$$

degenerates when $f(\phi)=0$. If $f(\phi) \leq 0$, is it in principle possible that also

$$
f_{1}(\phi) \equiv f(\phi)+\frac{3}{2}\left(\frac{d f}{d \phi}\right)^{2}
$$

vanish [6], which causes the effective coupling (3) to diverge if $\omega=1$ (the case considered by the authors of Ref. [1]). The set of points at which $f_{1}(\phi)=0$ constitutes a singularity in the transformation (5) that is necessary to bring the scalar field into canonical form in the Einstein frame.

The first kind of singularity is usually avoided by imposing that $f(\phi)>0$, which guarantees that gravity is attractive and that the graviton carries positive energy. However, the scalar may be allowed to assume values that make $f(\phi)$ negative, which are not 
a priori forbidden by the classical dynamics. This is the case, e.g., of the nonminimally coupled theory described by $f(\phi)=(8 \pi G)^{-1}-\xi \phi^{2}$ - in the literature one often encounters situations in which $G_{e f f} \equiv G\left(1-8 \pi G \xi \phi^{2}\right)^{-1}<0$ [7]. This regime is sometimes disguised by the fact that the field equations are written as

$$
G_{a b}=8 \pi G_{e f f} T_{a b}^{(e f f)}[\phi],
$$

where $T_{a b}^{(e f f)}[\phi]$ is an effective energy-momentum tensor for the scalar $\phi[8]$. When the time-time component of eq. (7) is considered, $G_{\text {eff }}$ is allowed to become negative but the Hamiltonian constraint is satisfied because the effective energy density of the scalar $T_{a b}^{(e f f)}[\phi] u^{a} u^{b}$ (where $u^{a}$ denotes the time direction) also becomes negative.

The singularity $f(\phi)=0$ in $G_{\text {eff }}$ corresponds to the loss of predictability at an hypersurface that is not hidden inside an event horizon. The Cauchy problem is well-posed in the Einstein frame, but not in the Jordan frame [10] and the vanishing of $f$ precludes the possibility of defining the Einstein frame metric (4). Although even in general relativity not every solution corresponds to a globally hyperbolic spacetime (e.g., $p p$-waves [11]), for many authors the idea of a naked Cauchy horizon is sufficiently unpleasant to require a priori $f(\phi)>0$. An added disadvantage of the $f=0$ singularity is that it invalidates the covariant and gauge-invariant analysis of cosmological perturbations [12] and the equations ruling the evolution of these perturbations become singular when $f \rightarrow 0$. Nevertheless, the dynamics of the unperturbed universe do not disallow values of $\phi$ such that $f(\phi)=0$. Abramo et al. have studied the singularities $f(\phi)=0$ and $f_{1}(\phi)=0$ in a Bianchi I model: both of them turn out to be true spacetime singularities with divergent Kretschmann scalar $R_{a b c d} R^{a b c d}[1,2]$.

Let us turn our attention now to the second kind of singularities $f_{1}(\phi)=0$. For this singularity to occur it must be $f(\phi) \leq 0$ and hence requiring $f(\phi)$ to always be positive eliminates this singularity also. Further, even if negative values of $f$ are permitted, the singularity $f_{1}(\phi)=0$ may be forbidden by the classical dynamics (this is the case, e.g., of closed or critically open Friedmann-Lemaitre-Robertson-Walker (hereafter "FLRW") universes under reasonable assumptions - see below). In addition to the divergence of the effective coupling (3) if $\omega=1$, the $f_{1}=0$ singularity in eq. (5) precludes the possibility of reformulating the scalar-tensor theory in the Einstein frame. This circumstance does not a priori imply any pathology in the dynamics, but the $f_{1}=0$ singularity leaves one uncomfortable for the following reasons:

1. The Cauchy problem is not well-posed in the Jordan frame [10].

2. In the quantization of linearized scalar-tensor gravity, it is the Einstein frame and not the Jordan frame metric perturbation that is identified with the physical 
graviton and carries positive energy [13]-[15]. Being unable to define the Einstein frame then means that one cannot quantize linearized gravity.

Abramo et al. $[1,2]$ have shown that $f_{1}(\phi)=0$ occurs unless two conditions are met: $f$ and $d f / d \phi$ simultaneously vanish and $f_{1}$ has as zeros only the zeros of $f$ - such a situation occurs if $f(\phi) \propto \phi^{2 n}$ and $V(\phi) \propto \phi^{2(2 n-1)}$. This situation is discarded as fine-tuned by Abramo et al. but it is not at all unphysical: this is the case of induced gravity with a massive scalar, described by $f(\phi)=\epsilon \phi^{2}$ and $V(\phi)=m^{2} \phi^{2} / 2$.

Finally, we show that the $f_{1}=0$ singularity is dynamically avoided in a closed or critically open FLRW universe under physically reasonable assumptions. Our argument generalizes to scalar-tensor gravity an argument previously given in Refs. [16, 17] for the special case of non-minimally coupled scalar field theory.

The Hamiltonian constraint in a FLRW universe,

$$
3 f\left(H^{2}+\frac{K}{a^{2}}\right)=\rho^{(m)}+\frac{\omega}{2}(\dot{\phi})^{2}+V-3 H \dot{f},
$$

where $\rho^{(m)}$ is the energy density of matter, can be rewritten as

$$
\left(H+\frac{\dot{f}}{2 f}\right)^{2}=\left(\frac{\dot{f}}{2 f}\right)^{2}+\frac{\omega(\dot{\phi})^{2}}{6 f}+\frac{\rho^{(m)}}{3 f}-\frac{K}{a^{2}}+\frac{V}{3 f} .
$$

Now let us assume that $f+\frac{3}{2}\left(\frac{d f}{d \phi}\right)^{2} \leq 0$ (which implies that $f \leq 0$ ) - then the first two terms on the right hand side add up to

$$
\begin{aligned}
& \left(\frac{\dot{f}}{2 f}\right)^{2}+\frac{\omega(\dot{\phi})^{2}}{6 f}=\frac{1}{6}\left(\frac{\dot{\phi}}{f}\right)^{2}\left[\omega f+\frac{3}{2}\left(\frac{d f}{d \phi}\right)^{2}\right] \\
& <\frac{1}{6}\left(\frac{\dot{\phi}}{f}\right)^{2}(\omega-1) f \leq 0,
\end{aligned}
$$

where the last inequality is satisfied if $\omega \geq 1$, as is usually assumed, and hence the first two terms on the right hand side of eq. (9) give non-positive contributions. Now, by assuming that $V \geq 0, K=0$ or +1 , and $\rho^{(m)} \geq 0$, one also obtains

$$
\left(\rho^{(m)}+V\right) \frac{1}{3 f}-\frac{K}{a^{2}} \leq 0
$$

with the equality holding only in Minkowski space. Hence the left hand side of eq. (9) is non-negative, while the right hand side is negative, an absurdity caused by assuming 
that $f_{1} \equiv f+\frac{3}{2}\left(\frac{d f}{d \phi}\right)^{2} \leq 0$. The assumptions used in our proof automatically guarantee that $f_{1}(\phi)>0$ and that the $f_{1}=0$ singularity is dynamically avoided.

The consideration of a FLRW universe is not very relevant before inflation because the initial conditions are likely to be anisotropic, and it is inflation that leads to a highly homogeneous and isotropic universe. However, a FLRW metric is mandatory in quintessence models of the universe during the present era of accelerated expansion, where the conditions of this comment apply.

Although the interest of Ref. [1] in singularities of scalar-tensor gravity arises from nonminimally coupled scalar field cosmology, $f=0$ singularities also appear in studies of wormholes with nonminimally coupled scalar fields ([18] and references therein). In the future it would be interesting to study the stability of wormhole solutions corresponding to $f=0$.

\section{References}

[1] L.R. Abramo, L. Brenig, E. Gunzig, and A. Saa, Phys. Rev. D 67, 027301 (2003).

[2] L.R. Abramo, L. Brenig, E. Gunzig, and A. Saa, Int. J. Theor. Phys. 42, 1145 (2003).

[3] K. Nordtvedt, Phys. Rev. D 169, 1017 (1968).

[4] This expression can also be derived from cosmological perturbation theory [5].

[5] B. Boisseau, G. Esposito-Farèse, D. Polarski and A.A. Starobinsky, Phys. Rev. Lett. 85, 2236 (2000).

[6] In $D>2$ spacetime dimensions the function $f_{1}$ is defined as

$$
f_{1}(\phi) \equiv f(\phi)+\frac{D-1}{D-2}\left(\frac{d f}{d \phi}\right)^{2} .
$$

[7] L.E. Gurevich, A.M. Finkelstein and V.A. Ruban, Astr. Sp. Sci. 22, 231 (1973); A.D. Linde, Sov. Phys. JETP (Lett.) 30, 447 (1980); A.A. Starobinsky, Sov. Astron. (Lett.) 7, 36 (1981); M.D. Pollock, Phys. Lett. B 108, 386 (1982); Y. Hosotani, Phys. Rev. D 32, 1949 (1985).

[8] There are three possible and physically inequivalent ways of writing the field equations for nonminimally coupled scalar fields, and care must be taken in choosing the appropriate one [9]. 
[9] S. Bellucci and V. Faraoni, Nucl. Phys. B 640, 453 (2002).

[10] P. Teyssandier and P. Tourrenc, J. Math. Phys. 24, 2793 (1983).

[11] R. Penrose, Rev. Mod. Phys. 37, 1 (1965).

[12] J.-C. Hwang, Class. Quant. Grav. 7, 1613 (1990); J.-C. Hwang, Phys. Rev. D 42, 2601 (1990); J.-C. Hwang, Class. Quant. Grav. 8, 195 (1990); J.-C. Hwang, Phys. Rev. D 53, 762 (1996); J.-C. Hwang, Class. Quant. Grav. 14, 1981 (1997); J.-C. Hwang, and H. Noh, Phys. Rev. D 54, 1460 (1996); H. Noh and J.-C. Hwang, Phys. Lett. B 515, 231 (2001).

[13] H.H. Soleng, Class. Quant. Grav. 5, 1501 (1988).

[14] Y.M. Cho, Class. Quant. Grav. 14, 2963 (1997).

[15] T. Damour and G. Esposito-Farèse, Class. Quant. Grav. 9, 2093 (1992).

[16] T. Futamase and K. Maeda, Phys. Rev. D 39, 399 (1989).

[17] L. Amendola, M. Litterio and F. Occhionero, Int. J. Mod. Phys. A 5, 3861 (1990).

[18] C. Barcelo and M. Visser, Class. Quant. Grav. 17, 3843 (2000). 